\title{
DICTIONARY-BASED EQUIVALENT SOURCE METHOD FOR NEAR-FIELD ACOUSTIC HOLOGRAPHY
}

\author{
Antonio Canclini, Massimo Varini, Fabio Antonacci, Augusto Sarti \\ Politecnico di Milano \\ Dipartimento di Elettronica, Informazione e Bioingegneria \\ Via Ponzio 34/5, 20133 Milano, Italy
}

\begin{abstract}
In this paper, we propose a modification of the standard Equivalent Source Method (ESM) for Near-Field Acoustic Holography (NAH). As in EMS, we aim at modeling the acoustic pressure radiated from a vibrating object, and its surface velocity, as the joint effect of a set of equivalent sources located within or close to the object itself. The estimation of the equivalent source strengths (weigths) comes from the solution of a highly ill-conditioned problem. Rather than solving this problem in the least-squares sense, we exploit the 3D model of the vibrating object, along with a rough estimate of its physical parameters, to restrict the space of the solutions. More specifically, we make use of Finite Element Analysis for populating a compressed dictionary of possible equivalent source weights. NAH is then approached by seeking a sparse linear combination of the entries of the dictionary. Experiments carried on a public database prove the effectiveness of the proposed technique, especially when the number of available microphones is limited, and in the presence of a significant level of measurement noise.
\end{abstract}

Index Terms - Near-field acoustic holography, Equivalent Source Method, Modal Analysis, Microphone arrays

\section{INTRODUCTION}

Near-field acoustic holography (NAH) enables modal analysis of vibrating objects by means of acoustic measurements. Microphones are positioned very close to the vibrating surface, in order to capture evanescent wave components of the radiated soundfield, which carry information about the surface velocity. The measured soundfield is typically referred to as acoustic hologram. Being contactless, NAH constitutes an effective alternative to vibrometric analysis by means of accelerometric sensors. For many tasks, indeed, contact-less is highly desirable, for instance when the object surface is delicate (e.g., fragile plates of historical musical instruments, thinly varnished objects, etc.). Furthermore, not charging the structure from an inertial point of view, the analysis can be performed also on very small objects. In many situations, NAH is also preferable over optical techniques (e.g., laser vibrometry), as no problems arise when light-reflecting surfaces are analyzed.

Many methods for NAH were proposed in the literature. Most of them are based on the inversion of the first Rayleigh's integral [1], which relates the normal velocity of a planar surface with the acoustic pressure generated by effect of vibration. This inverse problem is highly ill-conditioned, thus many regularization techniques have been investigated $[2,3,4]$. An interesting regularization method was

This work is supported by the Cultural District of the City of Cremona and by the CARIPLO Foundation. proposed in [5], where vibration modes of star-shaped planar plates are described by a linear combination of plane-waves components. Using this model, the solution to the NAH problem is approximated using a compressive sampling approach, finding a sparse solution in the space spanned by plane-wave basis functions. This technique was named Nearfield ACoustic HOlography with Sparse regularization (NACHOS) by its authors, which released an implementation in the NACHOS toolbox, along with the NACHOS database [6].

A class of more general methods for NAH is that based on the inversion of the Kirchhoff-Helmholtz $(\mathrm{KH})$ integral, which provides a relationship between the normal velocity of arbitrarily shaped surfaces and the near-field radiated soundfield. The discretization of the KH integral leads to the Boundary Elements Method (BEM) [7], useful to solve the forward problem, i.e. that of estimating the acoustic pressure given the knowledge of the normal velocity. The inverse problem can be handled by the Inverse BEM (IBEM), introduced to enable NAH for arbitrarily complex surface geometries [8]. The vibrating surface is approximated by means of convex polygons (typically triangles or rectangles), properly connected each other, all contributing to model the radiated soundfield.

Although leading to accurate results, the applicability of IBEM is severely limited by its extreme computational complexity. An interesting approximation of IBEM is the Equivalent Source Method (ESM) for NAH [9], originally introduced as the wave superposition method [10]. ESM models the radiated soundfield as being generated by a set of equivalent (virtual) sources located within (or in proximity of) the vibrating objects. ESM-based NAH operates in two steps. The first step involves the solution of an inverse problem to find the strengths (complex weights) of the equivalent sources that generate the measured acoustic pressure. Then, a suitable propagator function is applied to the equivalent sources, in order to infer the velocity on the surface of the vibrating source. The computational cost of ESM is therefore highly reduced compared to IBEM [11]. Unfortunately, ESM is dependent on the number and positioning of the equivalent sources, which highly influence the accuracy of the estimation. Some works in the literature attempted to derive rules and guidelines for the optimal positioning of equivalent sources (e.g., [12]), nevertheless this task still represents an open issue.

In this paper we propose a novel technique for NAH, which combines the flexibility of the ESM with the advantages of seeking the solution in a sparse domain. The proposed technique aims at being robust in those situations where it is not possible to use a large number of microphones and in the presence of measurement noise (e.g., interferences or residual reverberation when the measurement room is not perfectly anechoic). In particular, we propose a modification of the standard ESM scheme, restricting the ESM solution space to a suitable compressed dictionary. More specifically, given a 3D model 
(mesh) of the vibrating object, we predict the theoretical normal surface velocity by means of Finite Element Analysis (FEA) [13]. The theoretical velocity field is then back-propagated to the equivalent source positions to compute the corresponding weights. Several sets of source weights are collected by varying the physical parameters of the object material (which are unknown, in general), for each mode of vibration detected by FEA. The resulting sets of source weights are then processed through principal component analysis, in order to build a compressed dictionary. The sparse linear combination of equivalent source weights that best fits the measured acoustic hologram represents the ESM solution, which is finally forwardpropagated to the surface to estimate the real normal velocity. The proposed method was validated considering the aforementioned public NACHOS database, and compared with the NACHOS technique and the standard ESM. Experimental results confirm the effectiveness of the dictionary-based ESM approach.

The rest of the paper is structured as follows. Sect. 2 introduces some background on physical acoustics. The proposed solution to NAH is then described in Sect. 3. The experimental results are reported in Sect. 4, and Sect. 5 draws some final conclusions.

\section{BACKGROUND}

In this section we introduce the theoretical background at the base of the proposed NAH method. We start describing the KirchoffHelmholtz integral equation, which provides the relationship between the normal velocity and the acoustic pressure radiated from a vibrating surface. Then, we will outline the standard equivalent source method for NAH problems.

\subsection{Kirchhoff-Helmholtz integral}

Let us consider a vibrating object occupying a volume $V$ with an arbitrarily-shaped bounding surface $S$. We denote a point on $S$ with the position vector $\mathbf{s}$, and with $\mathbf{n}$ the unit vector normal to $S$ at $\mathbf{s}$. By applying the Green's theorem, the Fourier transform of the acoustic pressure at a point $\mathbf{r}$ outside the volume is given by the KirchhoffHelmholtz (KH) integral equation for exterior (radiation) problems [1], which can be expressed as [10]

$p(\mathbf{r}, \omega)=\int_{S} p(\mathbf{s}, \omega) \frac{\partial}{\partial \mathbf{n}} g_{\omega}(\mathbf{r}, \mathbf{s}) d \mathbf{s}-j \omega \rho_{0} \int_{S} v_{n}(\mathbf{s}, \omega) g_{\omega}(\mathbf{r}, \mathbf{s}) d \mathbf{s}$,

where $\omega$ is the angular frequency; $p(\mathbf{s}, \omega)$ is the Fourier transform of the surface acoustic pressure; $v_{n}(\mathbf{s}, \omega)$ is the surface normal velocity at $\mathbf{s}$; and $\rho_{0} \approx 1.2 \mathrm{~kg} / \mathrm{m}^{3}$ is the air mass density at $20^{\circ} \mathrm{C}$. The term

$$
g_{\omega}(\mathbf{r}, \mathbf{s})=\frac{1}{4 \pi} \frac{e^{-j \frac{\omega}{c}\|\mathbf{r}-\mathbf{s}\|}}{\|\mathbf{r}-\mathbf{s}\|}
$$

is the free-field Green's function, which describes acoustic wave propagation from $\mathbf{s}$ to $\mathbf{r}, c$ being the speed of sound. Equation (1) provides the integral relationship between the surface velocity and the radiated acoustic pressure, and constitutes the basis of the equivalent source method summarized in Section 2.2.

\subsection{Equivalent source method for NAH}

A complex acoustic radiator can be modeled as a superposition of elementary sources located within (or close to) the volume $V$ occupied by the radiator itself, and (1) can be reformulated as [10]

$$
p(\mathbf{r}, \omega)=j \omega \rho_{0} \int_{V} q(\mathbf{a}, \omega) g_{\omega}(\mathbf{r}, \mathbf{a}) d \mathbf{a},
$$

where $q(\mathbf{a}, \omega)$ represents the velocity flow of a source located at a point a within the volume $V$. For simplicity, we will refer to $q(\mathbf{a}, \omega)$ as a complex equivalent source weight. NAH can be approached in a simple and efficient way by discretizing (2) [9]. More specifically, we consider a finite set of $N$ equivalent sources located at $\mathbf{a}_{1}, \ldots, \mathbf{a}_{N}$ inside (or in the vicinity of) the vibrating object. The acoustic hologram, measured at $M$ positions $\mathbf{r}_{1}, \ldots, \mathbf{r}_{M}$, can be represented in matrix form as

$$
\mathbf{p}(\omega)=j \omega \rho_{0} \mathbf{G}_{p}(\omega) \mathbf{q}(\omega), \quad\left[\mathbf{G}_{p}(\omega)\right]_{m n}=g_{\omega}\left(\mathbf{r}_{m}, \mathbf{a}_{n}\right)
$$

where $\mathbf{q}(\omega)=\left[q\left(\mathbf{a}_{1}, \omega\right), \ldots, q\left(\mathbf{a}_{N}, \omega\right)\right]^{T}$ are the unknown equivalent source weights; $m=1, \ldots, M$; and $n=1, \ldots, N$.

On the basis of this representation, the NAH solution is computed in two steps. First of all, the equivalent source weights are estimated by inverting (3) in the least-squares sense, i.e.

$$
\hat{\mathbf{q}}(\omega)=\left[\hat{q}\left(\mathbf{a}_{1}, \omega\right), \ldots, \hat{q}\left(\mathbf{a}_{N}, \omega\right)\right]^{T}=\frac{1}{j \omega \rho_{0}} \mathbf{G}_{p}^{\dagger} \mathbf{p},
$$

where $\mathbf{G}_{p}^{\dagger}$ is the Moore-Penrose pseudo-inverse of $\mathbf{G}_{p}$. Then, the vibration mode at $\omega$ is given by the surface normal velocity at $B$ discrete points on the object surface, estimated as [9]

$$
\hat{\mathbf{v}}(\omega)=\mathbf{G}_{v}(\omega) \hat{\mathbf{q}}(\omega), \quad\left[\mathbf{G}_{v}(\omega)\right]_{b n}=\frac{\partial}{\partial \mathbf{n}_{b}} g_{\omega}\left(\mathbf{s}_{b}, \mathbf{a}_{n}\right)
$$

where $\mathbf{s}_{1}, \ldots, \mathbf{s}_{B}$ are the surface points coordinates, $b=1, \ldots, B$; $\mathbf{n}_{b}$ denotes the unit vector normal to the surface at $\mathbf{s}_{b}$.

\section{DICTIONARY-BASED EQUIVALENT SOURCE METHOD}

The NAH solution provided by (4) relies on the inversion of the system (3), which is in general highly ill-conditioned. This makes the standard ESM method very sensitive to the measurement noise present in the acoustic hologram $\mathbf{p}$ and highly dependent on the choice of the set of equivalent sources (i.e., number of sources and their positioning) [9]. As a consequence, although (2) is a valid and equivalent formulation of the $\mathrm{KH}$ integral (1), its discretization and the measurement noise may severely impact on the NAH solution.

In this section we propose a modification of the standard ESM methodology, which consists of two main novel contributions:

- computation of a dictionary of ideal equivalent source weights, to restrict the space of solutions provided by (4);

- inversion of (3) using LASSO regression [14], i.e. seeking a sparse solution among the entries of the dictionary.

Details about the creation of the dictionary will be given in Section 3.1, while the sparse regression will be introduced in Section 3.2.

\subsection{Dictionary creation}

Prediction of vibration modes via finite element analysis: FEA can be exploited for predicting the theoretical modes of vibration of an arbitrary surface, given a 3D mesh model of the object under analysis, the boundary conditions, and the set of the physical parameters of the material. Unfortunately, the latter can be only roughly estimated in most of the cases, due for instance to non-ideal characteristics and inhomogeneous behavior of the physical structure of the object. This lack of knowledge prevents an accurate prediction of the modes of vibration. Nevertheless, we can fruitfully use FEA to compute a variety of possible theoretical modes of vibrations for a 
given 3D mesh model, by varying the physical parameters in a wide range around the average values for a specific material.

For a generic orthotropic material (e.g., wood), for which the material and mechanical properties differ along three mutuallyorthogonal twofold axes of rotational symmetry, we must consider $U=10$ independent material parameters: three Young's moduli; three shear moduli; three Poisson's ratios; and the density. When considering an isotropic material (e.g., aluminum, steel), instead, its properties are constant along all the spatial directions, thus $U=4$ parameters are sufficient for its characterization. Let us denote the average values for a specific material with $\boldsymbol{\theta}=\left[\theta_{1}, \ldots, \theta_{U}\right]$. For each parameter, we uniformly sample the range $\left[\theta_{i}-\Delta \theta_{i}, \theta_{i}+\Delta \theta_{i}\right]$ at $K_{i}$ points. A total of $K=K_{1} \cdot K_{2} \cdot \ldots \cdot K_{U}$ combinations are thus considered to perform $K$ independent FEAs for a given 3D mesh. Each FEA detects a set of $Z$ eigensolutions corresponding to the vibration modes for a specific configuration of physical parameters. More specifically, FEA results in the following set of theoretical surface normal velocities, for $k=1, \ldots, K$ :

$$
\overline{\mathbf{v}}_{z}^{k} \triangleq \overline{\mathbf{v}}^{k}\left(\omega_{z}\right)=\left[\bar{v}_{n}\left(\mathbf{s}_{1}, \omega_{z}\right), \ldots, \bar{v}_{n}\left(\mathbf{s}_{B}, \omega_{z}\right)\right]^{T},
$$

where $\mathbf{s}_{1}, \ldots, \mathbf{s}_{B}$ are the $3 \mathrm{D}$ coordinates of the incenters of convex polygons that approximate the vibrating surface, $B$ being the total number of polygons; $z$ is the index of the vibration mode, and $\omega_{z}$ is the corresponding eigenfrequency.

Computation of equivalent source weights: A set of equivalent source weights corresponding to the theoretical vibration modes $\overline{\mathbf{v}}_{z}^{k}$ can be obtained by inverting the system (5) in the least-squares sense. Specifically, for each vibration mode $z=1, \ldots, Z$ and for each parameter configuration $k=1, \ldots, K$, we compute

$$
\overline{\mathbf{q}}_{z}^{k} \triangleq \overline{\mathbf{q}}^{k}\left(\omega_{z}\right)=\mathbf{G}_{v}^{\dagger}\left(\omega_{z}\right) \overline{\mathbf{v}}_{z}^{k} .
$$

Note that such a problem is, in general, ill-conditioned. We therefore resort to use a Tikhonov regularization scheme [15], by suitably tuning the regularization parameter using the L-curve method [16]. It is important to observe that ill-conditioning has limited effect on the solution in this case, as we rely on practically noiseless data $\overline{\mathbf{v}}_{z}^{k}$ that outcome from FEA.

Weights compression: In order to obtain a compact dictionary, and to reduce the risk of data overfitting, we exploit the redundancy contained in the sets of weights $\overline{\mathbf{q}}_{z}^{k}$. We do so by means of Principal Component Analysis (PCA) [17]. More specifically, for each vibration mode, we group the equivalent source weights in the matrix

$$
\overline{\mathbf{Q}}_{z}=\left[\overline{\mathbf{q}}_{z}^{1}, \overline{\mathbf{q}}_{z}^{2}, \ldots, \overline{\mathbf{q}}_{z}^{K}\right]
$$

on which PCA is performed. We then select the first $L_{z}$ loading vectors $\mathbf{w}_{z}^{1}, \ldots, \mathbf{w}_{z}^{L}$ representing at least $99 \%$ of the total data energy. The compressed source weights for the $z$ th mode are thus given as

$$
\mathbf{S}_{z}=\overline{\mathbf{Q}}_{z}\left[\mathbf{w}_{z}^{1}, \ldots, \mathbf{w}_{z}^{L_{z}}\right]
$$

By concatenating these terms for all the vibration modes, we obtain the compressed dictionary of equivalent source weights as

$$
\mathbf{D}=\left[\mathbf{S}_{1}, \mathbf{S}_{2}, \cdots, \mathbf{S}_{Z}\right] .
$$

\subsection{Near-field acoustic holography}

We are now ready to reformulate the NAH problem taking into account the dictionary (10). We first observe that, for the $z$ th vibration mode, the columns of $\mathbf{S}_{z}$ form an orthogonal basis for the vectors $\overline{\mathbf{q}}_{z}^{1}, \overline{\mathbf{q}}_{z}^{2}, \ldots, \overline{\mathbf{q}}_{z}^{K}$. We can therefore assume, for the $z$ th vibration mode, that a generic set of equivalent source weights $\mathbf{q}\left(\omega_{z}\right)$ can be approximated by linearly combining the columns of $\mathbf{S}_{z}$. When an arbitrary frequency $\omega$ is considered, however, no information about the index $z$ of the corresponding vibration mode is available. Therefore, we should consider the entire dictionary to model the equivalent source weights as $\mathbf{q}(\omega) \approx \mathbf{D} \boldsymbol{\gamma}$, where the vector $\boldsymbol{\gamma}$ contains the coefficients of the linear combination. Clearly, when $\omega=\omega_{z}$, the coefficient vector $\gamma$ is non-zero only for the entries associated to the $z$ th vibration mode, in order to activate only the columns of $\mathbf{D}$ corresponding to $\mathbf{S}_{z}$. This means that $\mathbf{q}(\omega)$ is obtained as a sparse linear combination of the columns of the compressed dictionary $\mathbf{D}$.

As we are working with FEA predictions of theoretical vibration modes, it is possible to further reduce the space of possible solutions by selecting a subset of the dictionary $\mathbf{D}$. For the $k$ th parameter configuration, $\boldsymbol{\theta}_{k}=\left[\theta_{1, k}, \ldots, \theta_{U, k}\right]$, FEA identifies the set of eigenfrequencies $\omega_{z, k}, z=1, \ldots, Z$, associated to all the vibration modes. By collecting the FEA results for $k=1, \ldots K$, it is possible to determine the set of eigenfrequencies detected for the $z$ th vibration mode, i.e. $I_{z}=\left\{\omega_{z, k}\right\}_{k=1}^{K}$. As a consequence, the $z$ th vibration mode is associated to a frequency in the range $F_{z}=\left[\min \left(I_{z}\right), \max \left(I_{z}\right)\right]$. Using this information, we can consider a reduced dictionary $\mathbf{D}_{\omega}$ formed by the terms $\mathbf{S}_{z}$ compatible with the analyzed frequency $\omega$. Specifically, $\mathbf{D}_{\omega}$ includes all the terms $\mathbf{S}_{z}$ such that $\omega \in F_{z}$. Therefore, we can finally express the equivalent source weights as

$$
\mathbf{q}(\omega) \approx \mathbf{D}_{\omega} \boldsymbol{\beta}
$$

where $\boldsymbol{\beta}$ is still a sparse coefficient vector, but shorter than $\boldsymbol{\gamma}$.

Inserting (11) into (3) leads to

$$
\mathbf{p}(\omega)=j \omega \rho_{0} \mathbf{G}_{p}(\omega) \mathbf{D}_{\omega} \boldsymbol{\beta} .
$$

The coefficient vector $\boldsymbol{\beta}$ can be estimated using a LASSO regularization scheme [14] to promote the sparsity of the solution, i.e.

$$
\hat{\boldsymbol{\beta}}=\underset{\boldsymbol{\beta}}{\operatorname{argmin}}\left(\left\|\mathbf{p}(\omega)-j \omega \rho_{0} \mathbf{G}_{p}(\omega) \mathbf{D}_{\omega} \boldsymbol{\beta}\right\|_{2}^{2}+\lambda\|\boldsymbol{\beta}\|_{1}\right),
$$

where the regularization parameter $\lambda$ is chosen through generalized cross-validation [18]. The surface velocity is finally estimated as

$$
\hat{\mathbf{v}}(\omega)=\mathbf{G}_{v}(\omega) \mathbf{D}_{\omega} \hat{\boldsymbol{\beta}}
$$

\section{EXPERIMENTAL VALIDATION}

Setup and database description: For the validation of the proposed method we used data extracted from the NACHOS database [6], considering measurements acquired over a thin rectangular aluminum plate of size $500 \times 400 \times 4 \mathrm{~mm}^{3}$ excited with a force hammer. The database provides the acoustic pressure measured by a rectangular array of 1920 microphones, regularly spaced on a grid positioned $20 \mathrm{~mm}$ over the vibrating plate. The groundtruth plate velocity, measured by means of a laser vibrometer, is also provided. Details about the acquisition setup can be found in [5].

The proposed dictionary-based equivalent source method (DESM) was tested considering the setup shown in Fig. 1. More specifically, we considered two rectangular microphone arrays of 32 and 64 elements, by sampling the grid of 1920 positions available from the NACHOS database. In both the cases, the microphones were selected to cover the whole area above the vibrating plate. The equivalent sources were placed on a plane at a distance of $20 \mathrm{~mm}$ under the bottom surface of the plate, disposed on a regular rectangular grid of size $24 \times 20$ points. 


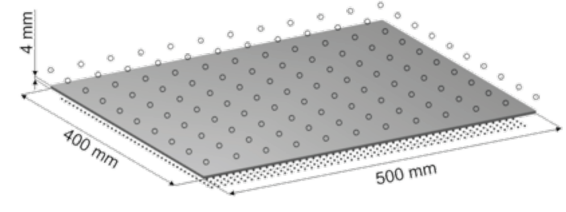

Fig. 1. Setup geometry: a regular grid of microphones (circles) is located on a plane above the aluminum plate; equivalent sources (dots) are positioned on a regular grid under the plate.

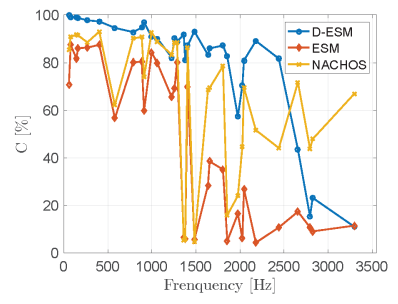

(a) 32 microphones

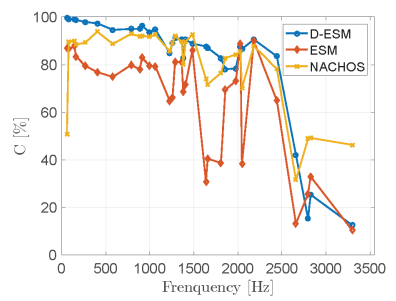

(b) 64 microphones
Fig. 2. Correlation score of vibration mode estimation.

The dictionary of equivalent source weights was populated following the procedure discussed in Section 3.1. As the aluminum is isotropic, we considered the four nominal physical parameters of the pure aluminum: density $\theta_{1}=2700 \mathrm{~kg} / \mathrm{m}^{3}$; Young's modulus $\theta_{2}=69 \mathrm{GPa}$; Poisson's ratio $\theta_{3}=0.34$; and shear modulus $\theta_{4}=25 \mathrm{GPa}$. We varied each parameter in the range $\pm 10 \%$ of the nominal value, uniformly sampling this range at 5 points, leading to a total of $K=5^{4}$ combinations. A triangular mesh model of the plate with 2860 elements was created, and FEA was performed using the built-in Matlab ${ }^{\circledR}$ Partial Difference Equation Toolbox.

Results: The proposed D-ESM method was tested to reconstruct 32 modes of vibration of the vibrating plate, in the range from $50 \mathrm{~Hz}$ to $3.3 \mathrm{kHz}$, in correspondence of the frequencies marked as relevant in the NACHOS database. D-ESM was compared to: i) the standard ESM, using the equivalent sources configuration described above; ii) the NACHOS technique, provided along with the NACHOS database; and iii) the groundtruth plate velocity.

To evaluate the accuracy of mode estimation, we consider the normalized correlation between the estimated plate velocity $\hat{\mathbf{v}}(\omega)$ and the groundtruth plate velocity $\mathbf{v}(\omega)$, computed as

$$
C(\omega)=\frac{\left|\hat{\mathbf{v}}^{T}(\omega) \mathbf{v}(\omega)\right|}{\|\hat{\mathbf{v}}(\omega)\| \cdot\|\mathbf{v}(\omega)\|} .
$$

The correlation scores for the considered frequency range are shown in Figures 2(a) and 2(b), relative to the 32- and 64-elements microphone arrays, respectively. We observe that D-ESM generally achieves better accuracy than both standard ESM and NACHOS techniques, for the two considered microphone configurations. At frequencies above $2.5 \mathrm{kHz}$ the accuracy of D-ESM tends to degrade. This is mainly due to the poor accuracy of FEA in predicting theoretical vibration modes at high frequencies, where a finer 3D mesh model would be required. Nevertheless, above $2 \mathrm{kHz}$ also ESM and NACHOS exhibit a pretty unstable behavior, especially when $32 \mathrm{mi}-$ crophones are considered, thus proving the effectiveness of the proposed technique when only a few microphones are available.

Examples of mode estimation at two selected frequencies are shown in Fig. 3, relative to NAH using 64 microphones. As expected from the correlation scores in Fig. 2(b), D-ESM accurately estimates the vibration patterns of the plate, clearly highlighting all

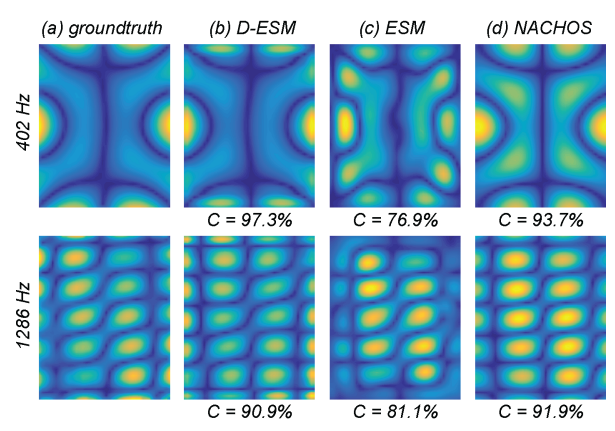

Fig. 3. Estimated surface velocity (magnitude).

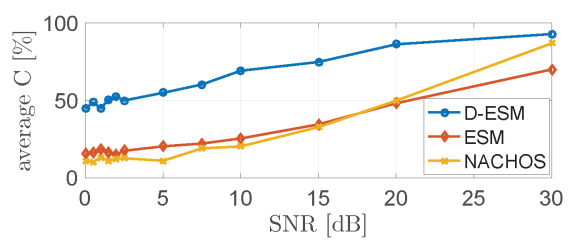

Fig. 4. Average correlation score vs SNR.

the nodal-lines and the anti-nodes. NACHOS introduces some small inaccuracies, while standard ESM presents more visible artifacts.

As a final experiment, we tested the robustness of the proposed $\mathrm{NAH}$ technique against the measurement noise. It is important to notice that the measurements in the database are not noiseless, as their acquisitions were carried in a non-ideal room [5]. Nevertheless, we injected additive white noise on the acoustic pressure measurements, which has to be considered as an additional synthetic noise component. The correlation scores were averaged in the range $[50,1500] \mathrm{Hz}$ where results are more stable for all the compared methods, and for both the array configurations. Results are reported in Fig. 4. Note that the reported signal-to-noise ratio (SNR) is only relative to the synthetic noise component, as the actual SNR of original data is not available. We observe that D-ESM turns to be the most robust method against the injected noise, maintaining its average accuracy around $50 \%$ even when the SNR is close to $0 \mathrm{~dB}$.

\section{CONCLUSIONS AND FUTURE WORKS}

In this work, we proposed a novel technique for NAH. The surface velocity of a vibrating object is estimated by means of the ESM, suitably modified in order to seek the solution as a sparse linear combination of the entries of a compressed dictionary. This dictionary is generated exploiting finite element analysis applied to a mesh model of the vibrating object. Experimental results show that the proposed D-ESM approach represents an effective alternative to the standard ESM when a few microphones are available in noisy scenarios. Moreover, D-ESM is generally more accurate than the state-of-the-art method NACHOS in analyzing a planar surface, at the expense of disposing of a 3D mesh model of the plate. Nevertheless, it is worth noticing that D-ESM do not poses any restrictions on the object, whose geometry can be arbitrary (either not star-shaped, nor planar, etc.). Note also that, for arbitrary surfaces, the availability of a mesh model is required also by state-of-the-art methods such as ESM and IBEM.

In the next few months we are going to set up experiments on complex surfaces. In particular we aim at using D-ESM to analyze the vibration modes of violin plates and bodies, whose surfaces exhibit holes and curvatures. 


\section{REFERENCES}

[1] E. G. Williams, Fourier acoustics: sound radiation and nearfield acoustical holography, Academic press, 1999.

[2] E. G. Williams, "Regularization methods for near-field acoustical holography," The Journal of the Acoustical Society of America, vol. 110, no. 4, pp. 1976-1988, 2001.

[3] Y. Kim and P. A. Nelson, "Optimal regularisation for acoustic source reconstruction by inverse methods," Journal of Sound and Vibration, vol. 275, no. 35, pp. 463-487, 2004.

[4] R. Scholte, I. Lopez, N. B. Roozen, and H. Nijmeijer, "Wavenumber domain regularization for near-field acoustic holography by means of modified filter functions and cut-off and slope iteration," Acta Acustica united with Acustica, vol. 94, no. 3, pp. 339-348, May/June 2008.

[5] G. Chardon, L. Daudet, A. Peillot, F. Ollivier, N. Bertin, and R. Gribonval, "Near-field acoustic holography using sparse regularization and compressive sampling principles," The Journal of the Acoustical Society of America, vol. 132, no. 3, pp. 1521-1534, 2012.

[6] G. Chardon, L. Daudet, A. Peillot, F. Ollivier, N. Bertin, and R. Gribonval, "Nachos database and toolbox," echange. inria.fr/nah.

[7] P. K. Banerjee and R. Butterfield, Boundary element methods in engineering science, vol. 17, McGraw-Hill London, 1981.

[8] W. A. Veronesi and J. D. Maynard, "Digital holographic reconstruction of sources with arbitrarily shaped surfaces," The Journal of the Acoustical Society of America, vol. 85, no. 2, pp. 588-598, 1989.

[9] Y. Zhang, F. Jacobsen, C. Bi, and X. Chen, "Near field acoustic holography based on the equivalent source method and pressure-velocity transducers," The Journal of the Acoustical Society of America, vol. 126, no. 3, pp. 1257-1263, 2009.
[10] G. H. Koopmann, L. Song, and J. B. Fahnline, "A method for computing acoustic fields based on the principle of wave superposition," The Journal of the Acoustical Society of America, vol. 86, no. 6, pp. 2433-2438, 1989.

[11] N. P. Valdivia and E. G. Williams, "Study of the comparison of the methods of equivalent sources and boundary element methods for near-field acoustic holography," The Journal of the Acoustical Society of America, vol. 120, no. 6, pp. 36943705, 2006.

[12] M. R. Bai, C. Chen, and J. Lin, "On optimal retreat distance for the equivalent source method-based nearfield acoustical holography," The Journal of the Acoustical Society of America, vol. 129, no. 3, pp. 1407-1416, 2011.

[13] O. C. Zienkiewicz and R. L. Taylor, The finite element method, vol. 3, McGraw-hill London, 1977.

[14] R. Tibshirani, "Regression shrinkage and selection via the lasso," Journal of the Royal Statistical Society. Series B (Methodological), pp. 267-288, 1996.

[15] A. N. Tikhonov and V. Y. Arsenin, Solutions of ill-posed problems, Winston, 1977.

[16] P. C. Hansen, "Analysis of discrete ill-posed problems by means of the 1-curve," SIAM review, vol. 34, no. 4, pp. 561580, 1992.

[17] H. Abdi and L. J. Williams, "Principal component analysis," Wiley Interdisciplinary Reviews: Computational Statistics, vol. 2, no. 4, pp. 433-459, 2010.

[18] G. H. Golub, M. Heath, and G. Wahba, "Generalized crossvalidation as a method for choosing a good ridge parameter," Technometrics, vol. 21, no. 2, pp. 215-223, 1979. 